Boletín de la Sociedad Geológica Mexicana Número Especial de Geología Urbana

Tomo LVIII, NúM. 2, 2006, P. 237-250

\title{
Mecanismos de fracturamiento de depósitos arcillosos en zonas urbanas. Caso de deformación diferencial en Chalco, Estado de México
}

\author{
Dora C. Carreón-Freyre ${ }^{1, *}$, Claudia M. Hidalgo-Moreno², Martín Hernández-Marín ${ }^{3}$ \\ ${ }^{1}$ Centro de Geociencias, Universidad Nacional Autónoma de México \\ Campus Juriquilla, Querétaro, México. \\ ${ }^{2}$ Colegio de Posgraduados. Campus Montecillo, Texcoco, México \\ ${ }^{3}$ Dept. of Geological Sciences Virginia Tech. USA. \\ *freyre@geociencias.unam.mx
}

\section{Resumen}

El fracturamiento de los depósitos arcillosos que rellenan las cuencas lacustres sobre las que se han desarrollado zonas urbanas se ha convertido en un problema que afecta a varias ciudades del centro de México. Los estudios realizados revelan la coexistencia de varios factores que determinan las características de diversos tipos de fracturamiento a diferentes escalas. Como factores principales, en este trabajo se analiza: la variación en la compresibilidad de los sedimentos que ocasiona deformaciones diferenciales y la extracción del agua subterránea que subyace a las secuencias lacustres y provoca la disminución de la presión de poro. La compresibilidad depende de la consolidación, que en mecánica de suelos se refiere a la expulsión de agua intersticial, y tiene como consecuencia la disminución de volumen y la subsidencia del terreno. Aún cuando la mayor reducción volumétrica se da en el sentido vertical, la consolidación de los materiales limo-arcillosos genera esfuerzos de tensión tanto verticales como horizontales. Considerando este fenómeno como determinante para la generación de fracturas, se llevó a cabo un análisis de las condiciones de deformación de una secuencia limoarcillosa integrando sus características estratigráficas y mecánicas. Se hace particular énfasis en la heterogeneidad mineralógica del material arcilloso para mostrar que ésta se puede traducir en variaciones de compresibilidad y generar fracturamiento por deformación diferencial. Como caso de estudio se presenta un análisis de propiedades geológicas y mecánicas de la secuencia lacustre de Chalco, para relacionar las diferencias de compresibilidad de los materiales con sus condiciones de formación. La historia paleoambiental determina el grado y tipo de cristalización de las diferentes fases minerales arcillosas, la granulometría y su historia de cargas litostáticas. En este trabajo se demuestra que el fracturamiento en depósitos fluvio-lacustres no es un fenómeno aleatorio, sino que depende de las propiedades geológicas de los materiales. Consideramos que el análisis de los parámetros que determinan la generación de fracturas debe llevar a la evaluación precisa de su propagación y del peligro que implica para la infraestructura urbana.

Palabras clave: Compresibilidad, Deformación, Depósitos lacustres, Arcillas, Fracturamiento.

Abstract

Fracturing of clayey lacustrine deposits below urban areas has become a major problem in several cities of central Mexico. The available data reveals the coexistence of several factors determining fracturing at different scales. As main factors we analyze in this study: the variation in compressibility of sediments causing differential deformation and withdrawal of groundwater causing a drop in pore pressure. Compressibility depends on consolidation, a term that in soil mechanics refers to the expulsion of interstitial water, and provokes volume decrease and land subsidence. Although major volume decrease occurs in the vertical scale, consolidation of silty clayey materials generates also horizontal tensile stresses. Considering that this factor can be determining to the generation of fractures, in this study we analyzed the deformational conditions of a silty clayey sequence integrating their stratigraphy and mechanical characteristics. A particular emphasis is made in the mineralogical heterogeneity of the clay fraction that can be related to compressibility variation and generate fracturing by differential deformation. As a study case we analyze the mechanical and 
geological properties of the Chalco lacustrine sequence in order to relate these properties with the differences in compressibility of materials. The paleoenvironmental history determines the type and degree of crystallization of different clay minerals, grain size, and history of lithostatic loads. Thus, the fracturing in fluvial lacustrine deposits is not a random phenomenon but is highly dependent of the geological properties of materials. We consider that the analysis of the parameters determining the generation of fracturing should permit a more precise evaluation of their propagation and of the implied danger to urban infrastructure.

Key words: Compressibility, Deformation, Lacustrine deposits, Clays, Fracturing.

\section{Introducción}

Algunas de las ciudades del centro del país establecidas en cuencas lacustres y que han sido afectadas por un problema generalizado de fracturamiento y/o subsidencia son: la Ciudad de México y zona conurbada, Querétaro, Morelia, San Luís Potosí, Aguascalientes, Celaya, Guadalajara, Salamanca, Abasolo, León e Irapuato. La mayor parte de estas ciudades se localiza sobre planicies horizontales que son la expresión en superficie de cuencas endorreicas rellenas con materiales sedimentarios fluvio-lacustres altamente heterogéneos en composición y estructura; y en la mayoría de los casos también de materiales volcánicos (principalmente lavas y materiales piroclásticos). Cabe remarcar que estas cuencas se encuentran delimitadas por el efecto combinado de edificios volcánicos y estructuras regionales.

El interés de estudiar los factores que influyen en la formación del fracturamiento en el relleno vulcano-sedimentario de estas cuencas es identificar los parámetros que permiten evaluar sus condiciones de generación y de propagación. Es claro que una mejor comprensión de este fenómeno llevará a una mejor cartografía del peligro que representa para la infraestructura urbana y permitirá establecer y validar una metodología sistemática de análisis para cuencas de origen fluvio-lacustre.

\section{Antecedentes}

El comportamiento mecánico y el fracturamiento del sistema lacustre de la Cuenca de México ha sido estudiado con relativo detalle por diferentes grupos desde la mitad del siglo anterior (Orozco y Figueroa, 1991), y la subsidencia en la Ciudad de México fue reportada incluso antes de iniciarse la extracción intensiva del agua subterránea (Gayol, 1929; citado en Mazari y Alberro, 1990). En 1952, Hiriart et al. mencionan a Nabor Carrillo (1947) como el primer científico que explicó “el mecanismo que regula la formación de grietas en el Valle de México", fenómeno que en aquella época ya se hacía notable. Las fracturas entonces observadas se presentaban en terrenos lacustres, aparecían en una época del año y en otra se extinguían. De igual manera, desde hace varias décadas ciertos aspectos de la relación entre la subsidencia regional, el fracturamiento y la extracción de agua subterránea fueron establecidos por varios autores (Zeeavert; 1953, Marsal y Mazari, 1959; Juarez Badillo, 1962; Juárez Badillo y Figueroa Vega, 1984; Figueroa Vega, 1989; Orozco and Figueroa, 1991 y Lugo-Hubp et al., 1991).

A partir de la década de los años setenta, el problema del fracturamiento de suelos recibió atención especial por parte de la Sociedad Mexicana de Mecánica de Suelos (SMMS). En una sesión del simposio "El subsuelo y la ingeniería de cimentaciones en el área urbana del Valle de México" (1978), se plantea que el origen de las fracturas en Naucalpan (La Florida y Echegaray) se relaciona con fallas por esfuerzo cortante asociadas a la consolidación diferencial del subsuelo y se reportan por primera vez las fracturas en las inmediaciones del cerro Xico, en Chalco. Durante el mismo simposio, Ellstein (1978) propone una teoría sobre el mecanismo de falla, en la que el estado de tensión del suelo genera una cuña triangular que se hunde entre los bloques delimitados por ambas fracturas. En esta discusión sobre el problema de fracturamiento de los sedimentos lacustres en la Ciudad de México, se retoman las hipótesis propuestas por Carrillo (1947), Marsal y Mazari (1959) y Zeevaert (1953) sobre la importancia del abatimiento de presión en los acuíferos en el origen del fenómeno.

Un segundo esfuerzo por la misma SMMS se llevó a cabo en 1991 con la publicación de un volumen especial sobre "Agrietamiento de Suelos". En este volumen Orozco y Figueroa (1991) hacen mención de los simposia internacionales sobre hundimientos del terreno (IASH: 1969, 1976, 1984 y 1991) en donde se presentan problemas semejantes en ciudades de Arizona, Idaho, California, Texas y Nevada en E.U.A. Resaltan los trabajos de Schumann y Poland (1969) que relacionan de manera directa las fisuras con abatimientos del agua subterránea en materiales arenosos; Holzer y Davis (1976) y Holzer (1984) hacen una clasificación de fracturas y proponen un método para estimar la profundidad de las mismas; Kreitler $(1976,1977)$ asocia la aparición de fallas a un sistema preexistente que actúa como barrera al flujo subterráneo formando escalones piezométricos. Larson (1984) describe las posiciones favorables para la aparición de grietas, Sandoval y Bartlett (1991) hacen una evaluación de probables hundimientos y agrietamientos en Arizona. En particular, el trabajo de Poland (1984) ha sido fundamental para el establecimiento de la relación cuantitativa entre la subsidencia y el abatimiento piezométrico, a través de la 
medición de la compresibilidad de los materiales en campo $\mathrm{y}$ en laboratorio.

Sobre la problemática en México, en el volumen de 1991de la SMMS se mencionan los casos de fracturamiento de las ciudades de Celaya (Trujillo Candelaria, 1989), Aguascalientes (Aranda y Aranda, 1985) que se asocian a abatimientos piezométricos de alrededor de $40 \mathrm{~m}$. Se mencionan también los casos de Toluca, Estado de México y Abasolo, Apaseo, Villagrán y Santa Cruz de Juventino Rosas en Guanajuato y se señala la influencia de la estratigrafía y del abatimiento piezométrico para el caso de la Ciudad de Querétaro (Trejo-Moedano y Baini, 1991). Estudios recientes como el de Juárez-Luna, et al. (2002) y Rojas et al. (2002) se enfocan a las relaciones numéricas o analíticas que permiten simular el fracturamiento bajo estados de esfuerzo específicos, pero no consideran las heterogeneidades de la secuencia ni la variación de sus propiedades mecánicas e hidráulicas en el espacio y en el tiempo, por lo que estas aproximaciones no pueden ser utilizadas para la evaluación de mecanismos de propagación. En este sentido, el trabajo de Álvarez-Manilla (2000) es precursor ya que introduce al modelo numérico la variación de la compresibilidad de los materiales a medida que desciende el nivel piezométrico del agua para un caso real en Querétaro.

\section{Discusión sobre los mecanismos de fracturamiento en materiales arcillosos}

En México se ha destacado la aplicación de modelos cuantitativos para el estudio de los mecanismos de fracturamiento bajo los siguientes aspectos: (1) Mecánica de fracturas con un criterio de propagación basado en el "factor de intensidad de esfuerzos crítico" y realización de modelaciones de propagación bajo presión hidráulica aplicando el método del Elemento Finito (Auvinet y Arias, 1991); (2) Teoría de flujo unidimensional hacia una batería de pozos (Juárez Badillo y Figueroa Vega, 1984 y Juárez Badillo, 1991); y (3) Cuantificación de las tensiones inducidas por el abatimiento del agua subterránea basada en la teoría de la elasticidad incluyendo fuerzas de filtración (Figueroa Vega, 1989; Alberro y Hernández, 1990).

A partir de un análisis de la literatura existente hasta entonces Orozco y Figueroa (1991) señalan que las fracturas aparecen en zonas de fuertes hundimientos (la mayoría se inician a profundidad y se propagan hacia la superficie) y de transiciones estratigráficas laterales. En la Figura 1 se muestra, de manera esquemática, la alta heterogeneidad de la secuencia estratigráfica en una cuenca fluvio-lacustre en zonas volcánicas relativamente recientes. La variabilidad

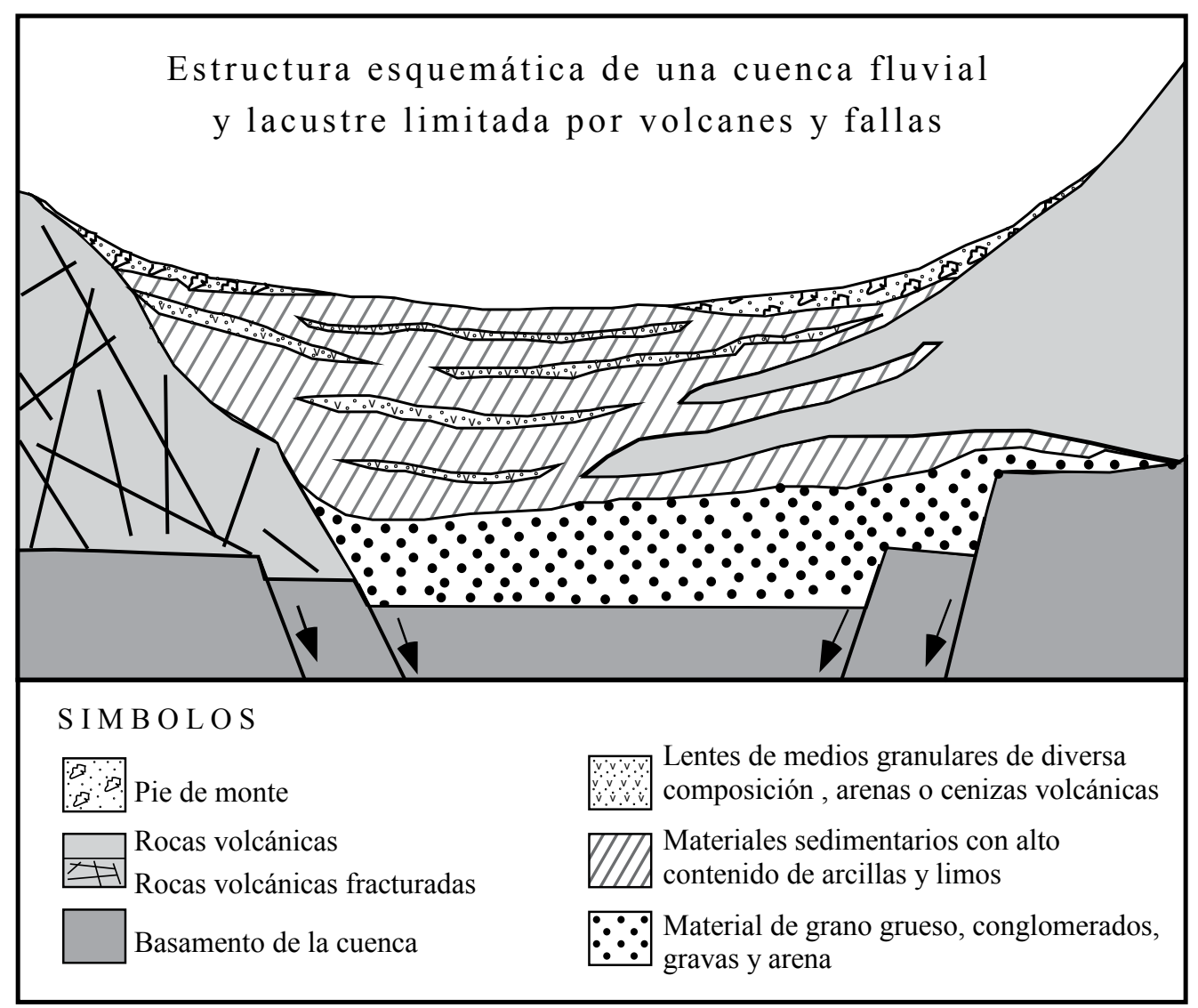

Figura 1. Esquema conceptual de sedimentación fluvio lacustre en una cuenca cerrada de origen volcánico, con actividad contemporánea a la sedimentación como es el caso de la Cuenca del Valle de México (Modificado de Carreón-Freyre et al., 2005a). 
lateral y en profundidad de la granulometría y permeabilidad del medio en este tipo de cuencas condiciona el comportamiento mecánico diferencial de los materiales y determina la distribución de gradientes hidráulicos críticos. Se puede establecer entonces que el fracturamiento de sedimentos está influenciado por la interacción entre los factores geológicos que condicionan sus propiedades y los factores disparadores que rompen el equilibrio mecánico del sistema.

\subsection{Factores geológicos que determinan la} susceptibilidad al fracturamiento

Las características geológicas de una secuencia sedimentaria, como el ambiente de depósito y estructuras pre-existentes en el basamento, son heterogeneidades determinantes para la formación y propagación de fracturas. La historia de cargas y variaciones de humedad durante la deposición de estos materiales determina la geometría de las primeras fracturas (p. ej. agrietamiento por desecación) que pueden modificar el estado local de esfuerzos y controlar la evolución de las fracturas alrededor de ellas (Tuckwell et al., 2003). Asimismo, la variación de la mineralogía del material arcilloso y de la permeabilidad de sedimentos y rocas se puede traducir en variaciones de compresibilidad y llevar al fracturamiento por deformación diferencial.

\subsubsection{Variaciones mineralógicas en una secuencia lacustre}

Los minerales arcillosos se forman por intemperismo, deposición o sedimentación, y en zonas volcánicas activas por alteración hidrotermal. De manera general, durante la alteración de rocas básicas, el sílice y el $\mathrm{Mg}$ son lixiviados, mientras que el $\mathrm{Fe}^{3+}$ y el $\mathrm{Al}$ se concentran en los horizontes superiores dando origen a la formación de suelos arcillosos residuales (Righi y Meunier, en Velde 1995). El material transportado continúa su evolución al ser depositado en un ambiente húmedo, parte del agua migra a través del suelo como drenaje interno, algunos minerales son disueltos y la solución se desplaza hacia las partes inferiores del perfil de suelo. El movimiento de elementos en el suelo está directamente relacionado con la estructura del suelo ya que las arcillas se acumulan en los horizontes medios del perfil reduciendo la permeabilidad de la parte superior en donde se pueden establecer condiciones de oxidación-reducción (Gama et al., 1998). En estas condiciones también se pueden formar algunos óxidos e hidróxidos de hierro que actúan como agregantes de las partículas del suelo. La neo-formación o formación autigénica de arcillas y otros minerales secundarios en la secuencia sedimentaria depende entonces de las concentraciones iónicas en el sistema del suelo, condiciones de Eh (oxidación-reducción) y pH (acidez-alcalinidad) y la cinética de las reacciones (Velde, 1995). A su vez, la cinética de las reacciones se relaciona directamente con las variaciones climáticas durante la historia de depósito de los materiales. Por ejemplo, en zonas con alternancia fuerte de climas y de estaciones secas-húmedas, en donde el lavado de cationes básicos $\mathrm{Ca}$ y $\mathrm{Mg}$ es mínimo, se forman arcillas esmectíticas (montmorillonita-beidellita-nontronita). También se favorece la formación de esmectitas en zonas de lago con drenaje restringido bajo condiciones salino-alcalinas (Hillier, en Velde, 1995).

La composición mineralógica de las arcillas de la Cuenca de México ha sido ampliamente documentada desde mediados del siglo pasado, debido principalmente a su heterogeneidad y a la complejidad de su comportamiento mecánico (fallamiento frágil en materiales plásticos, compresibles y con altos contenidos de agua). Los primeros trabajos reportaron composiciones contrastantes para los sedimentos de la Cuenca de México (Zeevaert, 1953; Marsal y Mazari, 1959; Mesri et al., 1976). A partir de sus propios análisis Peralta y Fabi (1989), concluye que la divergencia en los resultados reportados se debe a las variaciones de la mineralogía con la profundidad y describe una compleja estratificación microestructural de estos materiales y una composición microscópica semejante a un gel, similar a un material amorfo. A partir de entonces se han llevado a cabo estudios sobre la variación mineralógica de estos materiales con la profundidad (Díaz-Rodríguez et al., 1998, Mazari-Hiriart et al., 2000). Los resultados de la composición mineralógica de la fracción arcillosa reportados por diferentes autores son presentados en la Tabla $1 \mathrm{y}$, cuando se define, la profundidad y localización de las muestras estudiadas.

Cabe agregar que en secuencias sedimentarias como las México y Chalco, en donde la actividad volcánica es reciente y contemporánea a la deposición del relleno sedimentario, la rápida alteración de ceniza volcánica y pumicita genera suelos ricos en alofano e imogolita, minerales arcillosos de bajo orden similares a geles (Carreón-Freyre et al., 1998). Si las condiciones ambientales favorecen la deshidratación, estos materiales se transforman en gibbsita y halloysita (Righi y Meunier, en Velde, 1995). El alofano aunque amorfo a la Difracción de Rayos X, en microscopía electrónica (Wada, 1987) se presenta como partículas esféricas de aproximadamente $4 \mathrm{~nm}$ de diámetro, huecas e irregulares principalmente compuestas de sílice y aluminio. La imogolita tiene forma tubular, o de hilo, por lo que su frecuente asociación con el alofano da al suelo una estructura porosa abierta (Wesley, 2001) de ahí la elevada compresibilidad de estos materiales.

\subsubsection{Propiedades hidráulicas y mecánicas de los materiales arcillosos}

Existen numerosos trabajos de investigación sobre el estudio de la relación entre la mineralogía de los materiales arcillosos y sus propiedades mecánicas, las cuales dependen principalmente del contenido de agua y están directamente relacionadas con su baja permeabilidad. Por ejemplo, Ohstubo et al. (1983) correlacionan los 
Tabla 1. Características mineralógicas reportadas de la fracción arcillosa para la Cuenca de México.

\begin{tabular}{|c|c|}
\hline Autor & Composición mineralógica de la fracción arcillosa (\%) \\
\hline Zeevaert, 1953 & $\begin{array}{l}\text { Montmorillonita (20), cenizas, diatomeas y conchas microscopicas (65), feldespatos, } \\
\text { cuarzo y ferromagnesianos (15). Prof: } 0-25 \mathrm{~m}\end{array}$ \\
\hline Marsal y Mazari, 1959 & $\begin{array}{l}\text { Montmorillonita (13); ilitas (54); caolinita (4); dickita (2); halloysita (1); nontronita } \\
\text { (3); fosiles (3); } \mathrm{CaCO}_{3}(3-21) \text {, materia orgánica (1-7), no determinado (20). }\end{array}$ \\
\hline Lo, 1962 & $\begin{array}{l}\text { Amorfos-fragmentos de diatomeas, geles de silicoaluminato, mat. volc. alterado. } \\
\text { Montmorillonita de pobre cristalizacion, calcita (10), materia orgánica (4). } \\
\text { Prof: } 8 \text { m, Centro Cd. de México }\end{array}$ \\
\hline Leonards y Girault, 1961; Girault, 1964 & Alofanos (amorfos) en su mayoría, poca calcita, materia orgánica (8-10). \\
\hline Mesri et al. 1975 & $\begin{array}{l}\text { Interestratificado esmectita-silice(10), silice biogenetico y volcanico (90). } \\
\text { Prof. Aprox. } 15 \mathrm{~m}\end{array}$ \\
\hline Peralta y Fabi, 1989 & $\begin{array}{l}\text { Fracción arcillosa fina: montmorillonita, calcita, plagioclasa, probable cuarzo-alfa, } \\
\text { cristobalita, probable caolinita y/o clorita. } \\
\text { Arcillas (2-0.08 micras): esmectita (a veces Montmorillonita), clorita y/o caolinita } \\
\text { mal cristalizada, mica y nontronita } \\
\text { Prof: } 3.1 \text { a } 31.5 \text { m. Centro Cd. de México }\end{array}$ \\
\hline Warren y Rudolph, 1997 & $\begin{array}{l}\text { Alofano rico en sílice, montmorillonita ( } 30 \% \text { ), halloysita (solo a } 1.5 \mathrm{~m} \text { ), kaolinita e } \\
\text { illita (no se encontró imogolita, } \mathrm{pH}>5 \text { ) } \\
\text { Prof: } 0-10 \mathrm{~m} \text {, Norte de la Cd. de México. }\end{array}$ \\
\hline Diaz-Rodriguez et al., 1998 & $\begin{array}{l}\text { La fracción arcillosa a lo largo del sondeo varía de } 20 \text { a } 55 \% \text {. } \\
\text { Monmorillonita, illita y cristobalita. } \\
\text { polimorfos de sílice: ópalo biogénico, ópalo C-T y cristobalita } \\
\text { Prof: } 0-40 \text { m, Centro Cd. de México. }\end{array}$ \\
\hline Mazari-Hiriart et al., 2000 & $\begin{array}{l}\text { Corrensita (interestratificado clorita-esmectita) }(>90 \%) \text {, trazas de cristobalita, material } \\
\text { amorfo, halloysita }(5-10 \%) \text {. } \\
\text { Prof. } 14.9 \text { a } 75.5 \mathrm{~m} \text {, Centro Cd. de México }\end{array}$ \\
\hline
\end{tabular}

límites de consistencia (plasticidad) con la variación de la capacidad de retención de agua de las partículas de suelo en función de las características químicas de las arcillas y del agua intersticial. La relación entre la mineralogía de suelos arcillosos y el desplazamiento de agua durante el proceso de consolidación ha sido ampliamente discutida (Wesley, 2001; Saarenketo, 1998; Warren y Rudolph, 1997). Se pueden diferenciar tres tipos básicos de agua de poro en los materiales arcillosos: el agua intermolecular que forma parte de la estructura del alofano, el agua adsorbida fuertemente adherida a las partículas arcillosas principalmente esmectíticas y el agua libre que se desplaza más fácilmente entre los agregados, contactos intergranulares y/o microfracturas en la matriz arcillosa y que esta relacionada con la consolidación primaria de estos materiales.

En los materiales de la Cuenca de México se ha estudiado la influencia de la mineralogía en su plasticidad y compresibilidad (Mesri et al., 1976) y en su resistencia (Díaz-Rodríguez y Santamarina, 2001). También se ha caracterizado la mineralogía de secuencias arcillosas para estimar su comportamiento mecánico (Gutiérrez Castorena et al., 2005). De Pablo-Galán et al. (2001) hacen una medición sistemática de las variaciones mineralógicas y de su influencia en su viscosidad para dos niveles de profundidad, 26 y $60 \mathrm{~m}$, estableciendo un comportamiento diferencial no uniforme entre las muestras analizadas.
Actualmente se reconoce que, así como la mineralogía obedece a variaciones en las condiciones climáticas y depósito, la variación del comportamiento mecánico en una secuencia arcillosa obedece además a la búsqueda de equilibrio del sistema ante la influencia antropogénica. Sin embargo, existen pocos estudios que relacionen las condiciones geológicas con las variaciones mineralógicas, hidráulicas y mecánicas de secuencias arcillosas lacustres (Carreón-Freyre, 2005), y por lo tanto se conoce poco sobre la respuesta de estos sistemas y los mecanismos de propagación de las fracturas.

\subsection{Factores disparadores de fracturas}

El principal factor disparador de fracturas en sedimentos fluvio-lacustres es el desequilibrio mecánico, ya que las propiedades de estos sistemas pueden variar en tiempos muy cortos. Existen fenómenos naturales que actúan como mecanismos disparadores de fracturas, como son la actividad sísmica y las inundaciones causadas por lluvias torrenciales; sin embargo, los mecanismos más importantes son de origen antropogénico. El desequilibro mecánico inducido por sobrecarga estática (capacidad de carga para construcción) y dinámica (fatiga de los materiales en vías terrestres) y la despresurización del medio geológico debido a la extracción de los recursos naturales del subsuelo, ya 
sea agua, petróleo o gas, son los factores disparadores más importantes del fracturamiento en zonas urbanas.

Específicamente para el caso de la extracción de agua subterránea de acuíferos granulares, el decaimiento de la presión de poro propicia la compactación creando importantes esfuerzos de tensión verticales y horizontales (Carrillo, 1947; Holzer and Davis, 1976; Holzer, 1984; Figueroa Vega, 1989). Es importante remarcar que las variaciones espaciales y temporales en el descenso piezométrico dentro de una cuenca fluvio-lacustre pueden ser causadas por heterogeneidades a diferentes escalas: mineralógicas, estratigráficas o estructurales y la asociaciones de los diferentes factores determina la formación, el tipo y la propagación de las fracturas.

\subsection{Tipos generales de fracturas en secuencias fluvio-lacustres}

Los sistemas de fracturamiento se pueden estudiar de acuerdo a su tamaño y a la asociación de factores que les dan origen. Como los conceptos regional y local son relativos ya que dependen de la escala y del tipo de estudio, para este trabajo se asumen los siguientes criterios:

1. Las estructuras regionales son de dimensiones mayores que la zona urbana afectada. La irregularidad del basamento fracturado que subyace a las secuencias sedimentarias determina en gran medida la ubicación del fracturamiento que se propaga desde profundidad hacia las secuencias sedimentarias someras retomando planos de debilidad preexistentes, como es el caso de Morelia o de Querétaro (Rojas González et al., 2002, Carreón-Freyre et al., 2005a). En zonas urbanas afectadas por sistemas de falla regionales se debe considerar la asociación espacial de las mismas con variaciones estratigráficas, de manera que una falla pueden actuar como canal preferencial o como barrera de flujo formando gradientes piezométricos importantes (Kreitler, 1977, Carreón-Freyre et al., 2005b).

2. Como escala intermedia se considera a los sistemas de fracturamiento que afectan principalmente la parte superior de la secuencia sedimentaria fluvio-lacustre frecuentemente interestratificada con materiales piroclásticos y volcánicos. En las cuencas del centro del país los espesores totales de estas secuencias pueden alcanzar más de 500 metros, pero se considera solamente la parte superior (los primeros $200 \mathrm{~m}$ ) porque es la profundidad actual promedio de los niveles de extracción de agua subterránea. En ésta escala uno de los principales mecanismos de fracturamiento es la deformación diferencial, ya que los materiales presentan una distribución heterogénea de propiedades hidráulicas y mecánicas (Zeevaert, 1953; Ellstein A, 1978; Orozco y Figueroa, 1991, Carreón-Freyre et al., 2003). Considerar que el fracturamiento es sólo debido al abatimiento de los niveles piezométricos del agua subterránea implica una simplificación del fenómeno, ya que los abatimientos mayores no se relacionan de manera directa con los des- plazamientos verticales en las zonas de fracturamiento. Por ejemplo, en la Ciudad de Querétaro algunas zonas de mayor descenso piezométrico se localizan en el techo de la falla central que atraviesa la ciudad (Carreón-Freyre et al., 2006; Carreón-Freyre et al., 2005b). Para una adecuada evaluación de las condiciones de propagación del fracturamiento en ésta escala, se debe estudiar las variaciones verticales de las propiedades hidráulicas del sistema acuífero y las variaciones laterales de la compresibilidad de los materiales (Figueroa Vega, 1989; Carreón-Freyre et al., 2005a).

3. La escala local se refiere al fracturamiento en zonas restringidas y puede variar de unos cuantos a algunas decenas de metros (todavía se puede medir de manera directa las propiedades de los materiales); ésta es la escala en la que se lleva a cabo la mayor parte de los estudios de mecánica de suelos. Como ejemplos de fracturamiento local se tiene: (1) las fracturas anulares de tensión que se generan en las zonas transicionales de los piedemontes de lomerios (Lugo Hubp et al., 1991) y que se relacionan con deslizamientos gravitacionales; (2) las grietas superficiales generadas por evaporación-desecación en extensiones planas arcillosas, generadas por los cambios en las condiciones climáticas y en la presión de poro de las capas sedimentarias inferiores; y (3) las fracturas por tensión que se generan por las fuerzas de filtración ocasionadas por el bombeo localizado (JuárezBadillo, 1962; Alberro y Hernández, 1990).

\section{Resultados de un caso de estudio: Planicie lacustre de Chalco, Edo. de México}

4.1. Análisis de las condiciones de deposición de los sedimentos en el Valle de México

De acuerdo con Mooser (1975), el drenaje en el Valle de México se restringió hace 780000 años con la formación al sur de la Sierra Chichinautzin. A partir de entonces inicia la depositación de sedimentos fluvio lacustres inter-estratificados con materiales que registran diferentes periodos de intensa actividad volcánica en los alrededores del valle. Actualmente el Valle de México comprende tres sistemas de lagos: Zumpango-Xaltocan, Texcoco y Xochimilco-Chalco. La cuenca lacustre de Chalco se localiza hacia el SE de la Cuenca de la Ciudad de México de la que se separa geomorfologicamente por la Sierra de Santa Catarina (Figura 2). El sitio de estudio se ubica en la planicie lacustre hacia el margen parte norte de la cuenca en donde apareció hace aproximadamente 15 años una fractura de varias decenas de metros de longitud (Figuras 2 y 3 ). La secuencia cronológica del relleno sedimentario del lago de Chalco durante los últimos 34000 años, ha sido definida en varios trabajos a partir del estudio de diatomeas, polen, susceptibilidad magnética y edades ${ }^{14} \mathrm{C}$ (Caballero y Guerrero-Ortega, 1998 y Lozano-Garcia et al., 1993). Caballero y OrtegaGuerrero (1998) relacionan la presencia de "tefras" o capas de ceniza y/o lapilli con las variaciones en el registro de 


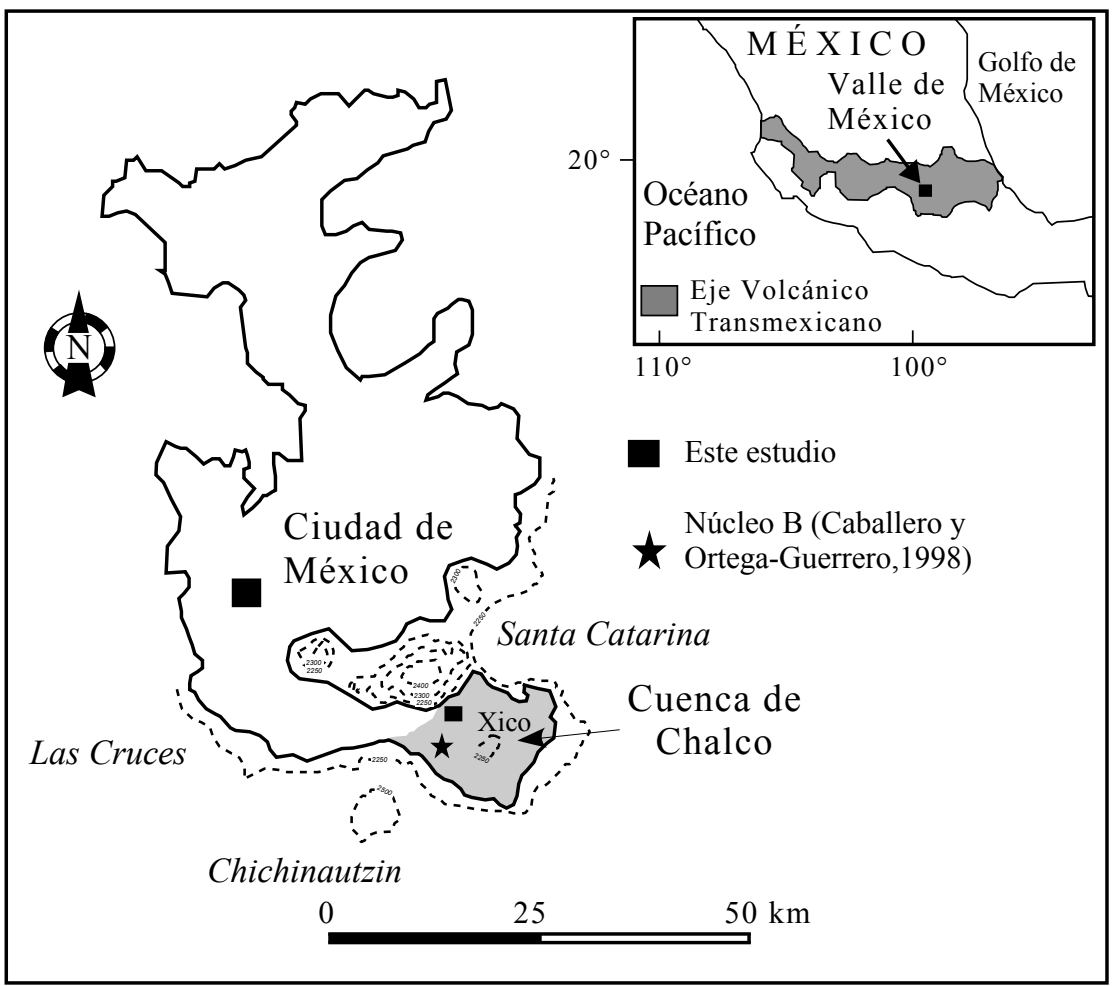

Figura 2. Localización del área de estudio en la Cuenca de Chalco (Modificado de Carreón-Freyre et al., 2003).

diatomeas y las atribuyen a cambios en las condiciones químicas y ambientales por el efecto de la caída de ceniza (Figura 4). En la Tabla 2 se presentan las condiciones de sedimentación definidas por Caballero y Guerrero-Ortega (1998). Para el estudio presentado en éste trabajo, se llevó a cabo un sondeo geotécnico con recuperación de muestra intacta hasta $15 \mathrm{~m}$ de profundidad con tubo de pared delgada de cuatro pulgadas. La caracterización física del la secuencia se efectuó de manera sistemática cada 25 cm y para la determinaciones mineralógicas y mecánicas se seleccionaron muestras representativas de los estratos identificados (Figura 5).

4.2. Caracterización mineralógica y física de la secuencia arcillosa de Chalco

\subsubsection{Análisis y caracterización en laboratorio}

Los resultados presentados comprenden la determinación de la mineralogía de los materiales arcillosos con Difracción de Rayos X efectuados en el laboratorio de Fertilidad de Suelos del Colegio de Posgraduados y en el Laboratorio de Fluorescencia de Rayos X del Instituto de Geología de la UNAM. La determinación de las propiedades índice y mecánicas de muestras inalteradas se llevó a cabo en el laboratorio de Geomecánica del Centro de Geociencias de la UNAM. La identificación de las unidades estratigráficas se llevó a cabo mediante la integración de todos los datos obtenidos laboratorio.
Los datos representativos de las propiedades físicas determinadas para cada estrato se presentan en la Tabla 3. En las primeras dos columnas se indica la profundidad del estrato identificado, la descripción por color y su siglas de clasificación de acuerdo al Sistema Unificado de Clasificación de Suelos (SUCS), en las siguientes columnas se presentan los valores de densidad de sólidos (Ss), granulometría (porcentajes de arena, limo y arcilla), contenido de agua gravimétrico natural, límites de consistencia o de Atterberg (Limite Líquido y Limite Plástico) y el contenido de materia orgánica. En la Tabla

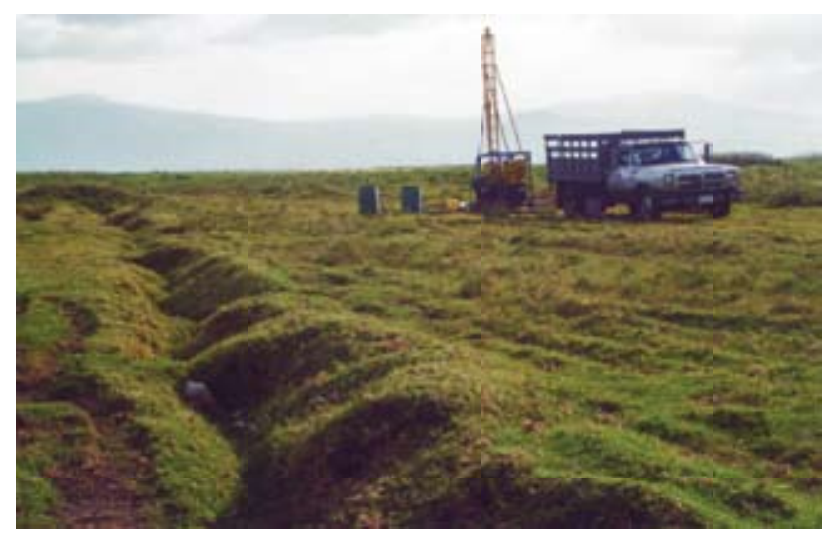

Figura 3. Fotografía de la planicie lacustre de Chalco. En primer plano se observa una fractura de varias decenas de longitud en la zona de estudio con orientación N10E. 


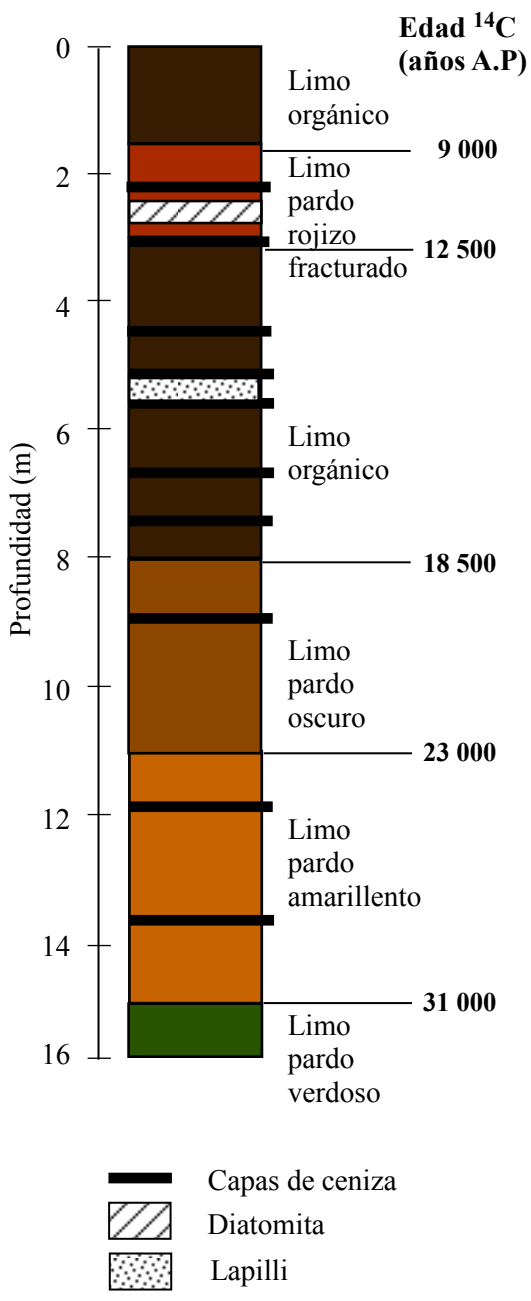

Figura 4. Secuencia estratigráfica y edad de radiocarbono del sondeo B, Cuenca de Chalco (modificado de Caballero y Ortega-Guerrero, 1998).

4 se presenta la variación en profundidad de las fases mineralógicas principales de los materiales arcillosos. Las fases mineralógicas con propiedades físicas y mecánicas más complejas son las esmécticas y el alofano. Las primeras se encontraron representadas por la presencia de montmorillonita con diferentes grados de cristalización. Este mineral es característico de zonas de lago con drenaje restringido bajo condiciones salino-alcalinas (Hillier en Velde, 1995) como las que prevalecieron en el lago de Chalco hace aproximadamente 30,000 años de acuerdo a Caballero y Guerrero-Ortega (1998). En este mismo ambiente se presenta la acumulación de óxidos de Fe, también reportado y cuando el hierro se reduce (colores verdosos) se vuelve soluble, se desplaza y se forma un complejo arcilloso interestratificado (Carreón et al., 1998). Las variaciones mineralógicas de los materiales estudiados pueden ser estimadas de manera indirecta mediante la determinación de los límites de consistencia Líquido (LL) y Plástico (LP). En un primer tiempo se determinaron los limites en condiciones de humedad natural y después dejándolo secar, de acuerdo con la norma ASTM D431895a. Las diferencias de los valores de índice de plasticidad (IP=LL-LP) obtenidos se presentan en la Tabla 5. Como se puede observar, existe una importante disminución en la magnitud del índice de plasticidad de un material arcilloso cuando se deja secar que varía de acuerdo con las características mineralógicas y grado de cristalización de las arcillas. Esto es debido a que las diferentes fases mineralógicas arcillosas retienen agua intermolecular o interparticular bajo diferentes condiciones (Saarenketo, 1998). La muestra de $1.8 \mathrm{~m}$ de profundidad con mayores contenidos de alofano presenta una variación mayor.

Consideramos que el comportamiento mecánico de los materiales estudiados está determinado por la presencia de montmorillonita con diferentes grados de cristalización, alofano y óxidos de hierro en condiciones variables de oxidación y reducción. Una de las propiedades mecánicas especialmente sensible a las diferentes capacidades de retención de agua de cada fase mineral es la compresibilidad, que se caracterizó con detalle en la secuencia.

\subsubsection{Descripción de la secuencia estratigráfica}

Un registro de la secuencia sedimentaria analizada se presenta en la Figura 5. Si se compara esta columna con la secuencia de la Figura 4 (definida por Caballero y Ortega-Guerrero, 1998), se observa una gran similitud en las variaciones de color de los limos arcillosos de la secuencia, las variaciones de espesor se deben a que un sondeo fue realizado en el centro de la cuenca y el de este trabajo en una zona marginal. La diferencia más evidente es la secuencia pardo-amarillenta correspondiente a las condiciones pantanosas que normalmente están ausentes en los márgenes de este tipo de cuenca. La secuencia arcillosa de la Figura 5 se puede dividir, para fines de caracterización geomecánica, en cuatro estratos de abajo hacia arriba. Se presenta entre paréntesis las condiciones de formación y el equivalente al tiempo de depositación para estratos semejantes definidos por Caballero y Ortega-Guerrero, (1998):

- Estrato de color gris oliva de los 15 a los $9 \mathrm{~m}$, con algunos horizontes de materia orgánica o de arena y en ocasiones con raíces o pequeñas grietas (lago de profundidad moderada, > 30,000 años AP; Antes del Presente.);

- Estrato de color pardo oscuro de 9 a $6 \mathrm{~m}$, con capas delgadas de color pardo amarillento y zonas enriquecidas de materia orgánica, inicia después del depósito de una capa de arena (o tefra) y presenta condiciones de oxidación en la parte superior (pantano, entre 30,000 y 20,000 años AP);

- Estrato de color negro de 6 a $3 \mathrm{~m}$ con una capa gruesa de material piroclástico a los cuatro metros, culmina con una capa de ceniza de aproximadamente $30 \mathrm{~cm}$ de espesor. Se observan además varias capas y/o lentes de ceniza-arena en la secuencia (lago de agua dulce con abundante vegetación y actividad volcánica contemporánea, entre 20,000 y 12,000 años $\mathrm{AP}$ ).

- Capa color gris pardo de diatomitas de $40 \mathrm{~cm}$ de espesor 
Tabla 2: Cronología y condiciones de sedimentación de la cuenca de Chalco (datos tomados de Caballero y Ortega-Guerrero, 1998).

\begin{tabular}{|c|c|c|}
\hline $\begin{array}{c}\text { Periodo } \\
\text { (Años A.P.) }\end{array}$ & Condiciones de sedimentación & $\begin{array}{l}\text { Unidad sedimentaria } \\
\text { Profundidad (m) }\end{array}$ \\
\hline $34,000-31,000$ & Lacustre, alcalinas y salinas, profundidad moderada & $\begin{array}{l}\text { Color pardo-verdoso } \\
15.75 \mathrm{~m}\end{array}$ \\
\hline $31,000-23,000$ & Pantano & $\begin{array}{l}\text { Color pardo-amarillento } \\
15.75-11 \mathrm{~m}\end{array}$ \\
\hline $23,000-18,500$ & $\begin{array}{l}\text { Lacustre, aguas dulces con profundidad moderada } \\
\text { con abundante vegetación }\end{array}$ & $\begin{array}{l}\text { Color pardo y pardo oscuro, } \\
11 \text { y } 8 \mathrm{~m}\end{array}$ \\
\hline 18,500 y 14,500 & $\begin{array}{l}\text { Palustre, fluctuaciones aguas ácidas y neutras } \\
\text { (dulces) Intensa actividad volcánica }\end{array}$ & $\begin{array}{l}\text { Limos orgánicos oscuros con capas de ceniza y lapilli } \\
8 \text { y } 3 \mathrm{~m}\end{array}$ \\
\hline 14,500 y 12,500 & $\begin{array}{l}\text { Aporte de sedimentos } \\
\text { Zona de bosque }\end{array}$ & \\
\hline $\begin{array}{l}12,500 \text { y } 9,000 \\
\text { (Pleistoceno) }\end{array}$ & $\begin{array}{l}\text { Incremento de humedad y los máximo desarrollo de } \\
\text { bosques }\end{array}$ & $\begin{array}{l}\text { Color gris, diatomitas, ceniza negra rellena fisuras } 3 \mathrm{~m} \text {, } \\
\text { Limo pardo rojizo con pómez, }\end{array}$ \\
\hline $\begin{array}{l}9,000 \text { y } 3,000(?) \\
\text { (Holoceno) }\end{array}$ & Pantano somero salino-alcalino & $\begin{array}{l}\text { capas de limo orgánico oscuro con fragmentos alterados } \\
\text { de pómez }\end{array}$ \\
\hline 3,000 y 1,000 & $\begin{array}{l}\text { Fluctuaciones entre lacustre, agua dulce, y pantano } \\
\text { alcalino. }\end{array}$ & \\
\hline
\end{tabular}

que indican una elevación temporal de nivel del agua y disminución de actividad volcánica (entre 12,000 y 9000 años, AP);

- Estrato de color pardo oscuro y rojizo, desde 2.61 a 0.80 $\mathrm{m}$ aproximadamente, con capas con grietas en algunas partes y lentes arenosos (lago somero-pantano salinoalcalino, 9,000-3,000 años AP).

4.3. Análisis de compresibilidad y condiciones de flujo en la secuencia de Chalco

De la secuencia estudiada (Figura 5) se seleccionaron muestras representativas de cada estrato para la realización de pruebas de compresibilidad que se llevaron a cabo con un consolidómetro computarizado con monitoreo de la presión de poro (Norma ASTM D2435-96). Las condiciones de realización de estas pruebas y la interpretación de los resultados de disipación de presión de poro para los diferentes tipos de materiales son discutidas por Hernández y Carreón-Freyre (2002). En la Tabla 6 se presentan valores representativos de los principales parámetros de compresibilidad para los estratos definidos en el inciso anterior. El grado de saturación de los materiales varía de 90 a 100 $\%$ en toda la secuencia por lo que se considera aplicable la teoría de consolidación de Terzaghi. El esfuerzo vertical efectivo $\left(\sigma_{\mathrm{v}}{ }_{\mathrm{v}}\right)$ se calculó considerando una profundidad de $1.8 \mathrm{~m}$ del nivel piezométrico en las arcillas (medida en campo), la carga de preconsolidación (Pc) se determinó utilizando el método de Casagrande y el Índice de Compresibilidad Cc se determinó en el tramo virgen de la curva de compresibilidad. Para fines comparativos se presenta también la variación de relación de vacíos (ei-ef) para las condiciones de carga aplicada (Pap) correspondientes al tramo de determinación de Cc. Como se puede observar en la Figura 6, la variación de Cc con la profundidad no corresponde directamente con la carga litostática, es decir la compresibilidad no es menor para mayor carga litostática ni mayor en superficie como se simplifica normalmente, lo que muestra su dependencia de las propiedades geológicas de los materiales.

De acuerdo con los valores reportados para las arcillas de la Cuenca de México (Marsal y Masari, 1959, Mesri et al., 1976; Díaz-Rodríguez et al., 1998), las propiedades de los materiales estudiados en Chalco están por debajo de las características geotécnicas típicas, que incluyen elevadas relaciones de vacíos (6 a 11) y de contenido de agua ( $>$ de $400 \%$ ) y valores de Índice de Compresión entre 3 y 6 . Aun cuando ambas cuencas tienen historias geológicas similares, su historia de cargas y la mineralogía de sus sedimentos pueden tener diferencias importantes relacionadas con las condiciones del depósito.

La variación en las curvas de compresibilidad de las muestras representativas de los diferentes estratos muestra la influencia de la fase mineralógica predominante (esmectitas, interestratificados o alofano), de las condiciones de oxidación o reducción del Fe presente (que se indica con los colores rojizo o verdoso respectivamente) y la presencia de microgrietas o lentes arenosos que aumentan la permeabilidad del suelo (Figura 7). Las muestras $\mathrm{CH} 1$, $\mathrm{CH} 2, \mathrm{CH} 3$ y CH4 (la muestra $\mathrm{CH} 5$ es remoldeada) no tienen una estructura estable ya que contienen cantidades variables de alofano, materiales arcillosos interestratificados y esmectita mal cristalizada, y presentan una compresibilidad altamente variable que no se puede relacionar de manera directa con la relación de vacíos debido probablemente a 


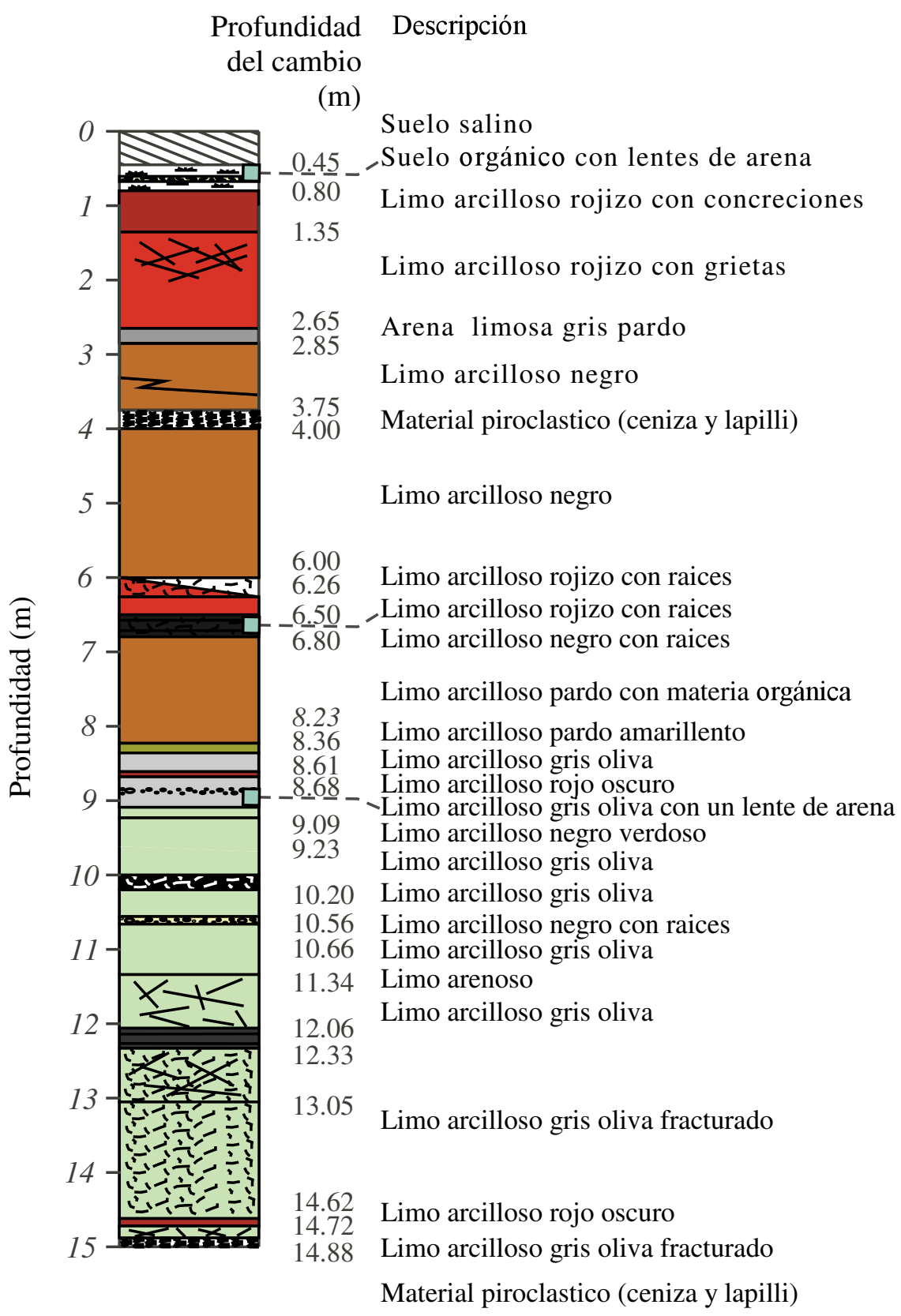

Figura 5. Diagrama de la secuencia estratigráfica estudiada en el norte de la planicie lacustre de Chalco. Identificación y caracterización de un sondeo de recuperación continúa de muestra intacta.

las diferentes condiciones del agua en una estructura cristalina incipiente. De acuerdo con Warren y Rudolph (1997) y Wesley (2001), la presencia predominante de alofano en los sedimentos lacustres es responsable de muchas de sus características especiales, como alta porosidad, alto contenido de agua y una Capacidad de Intercambio Catiónico (CEC) extremadamente dependiente del $\mathrm{pH}$. Las características generales de la arcilla alofánica comprenden una elevada superficie específica $\left(1000 \mathrm{~m}^{2} / \mathrm{g}\right)$ y condiciones de formaciones con $\mathrm{pH}$ entre 5 y 7 (Wada, 1987). La muestra CH6 presenta el comportamiento normal de un limo arcilloso lacustre ligeramente consolidado, mientras que las muestras $\mathrm{CH} 9$ y $\mathrm{CH} 10$, presentan el comportamiento de típico de arcillas esmectíticas mejor cristalizadas (alta relación de vacíos y alta compresibilidad). A partir de las pruebas de consolidación se calcularon los coeficientes de permeabilidad para diferentes materiales que, de manera general, varían entre $10^{-8} \mathrm{y}^{1} 0^{-7} \mathrm{~ms}^{-1}$. Los valores obtenidos no muestran una tendencia definida de variación para los diferentes tipos de materiales, y más bien parecen depender solamente del grado de fracturamiento y de la presencia de material arenoso. Marsal y Mazari (1959) reportan que la permeabilidad de estos materiales en laboratorio varía entre $10^{-9}$ y $10^{-10} \mathrm{~ms}^{-1}$. 
Tabla 3. Propiedades índice y clasificación de la secuencia estudiada en Chalco de acuerdo al Sistema Unificado de Clasificación de Suelos (SUCS). Variación en profundidad. Ss, densidad de sólidos; C-M-S; porcentajes de arcilla, limo y arena; w \%; contenido de agua natural, LL-LP; límite liquido y limite plástico; MO \%, contenido de materia orgánica.

\begin{tabular}{|c|c|c|c|c|c|c|}
\hline $\begin{array}{c}\text { Prof. } \\
\text { (m) }\end{array}$ & Color - SUCS & $\begin{array}{c}\text { Ss } \\
\mathrm{g} / \mathrm{cm}^{3}\end{array}$ & $\begin{array}{c}\text { C-M-S } \\
\%\end{array}$ & $\begin{array}{l}\mathbf{w} \\
\%\end{array}$ & LL-LP & $\begin{array}{r}\text { MO } \\
\%\end{array}$ \\
\hline 1.40 & $\begin{array}{l}\text { Rojo oscuro } \\
\text { OH-MH }\end{array}$ & 1.92 & $18-46-36$ & 292 & $247-190$ & 5.0 \\
\hline 1.84 & $\begin{array}{l}\text { Rojo oscuro grietas } \\
\text { OH-MH }\end{array}$ & 2.13 & $18-61-21$ & 290 & $364-211$ & 7.0 \\
\hline 2.44 & $\begin{array}{l}\text { Rojo oscuro } \\
\text { OH-MH }\end{array}$ & 2.09 & $27-34-39$ & 88 & $141-92$ & 6.5 \\
\hline 3.75 & Negro OH-MH & 1.53 & $24-60-16$ & 91 & $164-102$ & 11.8 \\
\hline 7.39 & $\begin{array}{l}\text { Pardo oscuro } \\
\text { OH-MH }\end{array}$ & 1.75 & $21-71-8$ & 154 & $260-162$ & 10.5 \\
\hline 9.2 & Gris oliva $\mathrm{CH}$ & 2.06 & $27-63-10$ & 199 & $151-59$ & 8.5 \\
\hline 11.9 & Gris oliva OH-MH & 2.25 & & 195 & $215-91$ & 7.1 \\
\hline 12.7 & $\begin{array}{l}\text { Gris oliva grietas } \\
\text { OH-MH }\end{array}$ & 2.16 & $10-77-13$ & 234 & $200-100$ & 6.5 \\
\hline 14.8 & Gris oliva OH-MH & 2.06 & & 204 & 215-104 & 6.7 \\
\hline
\end{tabular}

Los resultados presentados muestran que la compresibilidad refleja la influencia de la estructura de la matriz del suelo y de su relación con las condiciones de flujo de agua (cabe recordar que las variaciones estructurales en el suelo dependen de las condiciones climáticas de formación, mineralogía e historia de cargas). El análisis de la relación de vacíos y cargas de preconsolidación, permite establecer diferencias entre la consolidación de los materiales sujetos a periodos de desecación y de los materiales con algún tipo de agrietamiento o material arenoso inter-estratificado. Asimismo, las importantes variaciones de compresibilidad en la secuencia se pueden utilizar para validar el mecanismo de fracturamiento por deformación diferencial.

Tabla 4: Caracterización mineralógica de la secuencia estudiada en Chalco mediante Difracción de Rayos X. Variación en profundidad.

\begin{tabular}{clll}
\hline $\begin{array}{c}\text { Prof. } \\
(\mathbf{m})\end{array}$ & \multicolumn{1}{c}{ Estrato } & $\begin{array}{l}\text { Fase mineralo- } \\
\text { gica principal }\end{array}$ & \multicolumn{1}{c}{ Otros } \\
\hline $0.80-2.60$ & $\begin{array}{l}\text { Limo pardo } \\
\text { rojizo }\end{array}$ & Alofano & $\begin{array}{l}\text { Presencia incierta de } \\
\text { Montmorillonita }\end{array}$ \\
$3.40-5.40$ & $\begin{array}{l}\text { Limo orgánico } \\
\text { oscuro }\end{array}$ & $\begin{array}{l}\text { Alofano en alto } \\
\text { contenido }\end{array}$ & $\begin{array}{l}\text { Montmorillonita po- } \\
\text { bremente cristalizada }\end{array}$ \\
$6.50-8.60$ & $\begin{array}{l}\text { Limo arcilloso } \\
\text { pardo oscuro }\end{array}$ & Alofano & $\begin{array}{l}\text { Feldespatos } \\
\text { Montmorillonita po- } \\
\text { bremente cristalizada }\end{array}$ \\
$9.35-12.35$ & $\begin{array}{l}\text { Arcillas } \\
\text { Gris-verde } \\
\text { oliva }\end{array}$ & $\begin{array}{l}\text { Alofano } \\
\text { Montmorillonita } \\
\text { confirmada }\end{array}$ & $\begin{array}{l}\text { Montmorillonita po- } \\
\text { bremente cristalizada }\end{array}$ \\
$12.35-14.30 \begin{array}{l}\text { Limo arcilloso } \\
\text { Gris-verde } \\
\text { oliva }\end{array}$ & $\begin{array}{lll}\text { Alofano } \\
\text { Feldespatos }\end{array}$ \\
\hline
\end{tabular}

\section{Conclusiones}

En este trabajo se discuten los mecanismos de fracturamiento en secuencias fluvio-lacustres y se hace una diferencia entre los factores geológicos que propician la generación de fracturas y los factores disparadores, principalmente antropogénicos. Es importante considerar que las características estratigráficas de los suelos y la disminución de la presión de poro en una secuencia fluvio-lacustre no presentan una distribución homogénea, lo que se observa en el comportamiento mecánico de los materiales y se traduce en deformaciones diferenciales. Como un caso de estudio se presenta el análisis detallado de las propiedades mineralógicas, físicas y mecánicas de la secuencia lacustre somera de Chalco (hasta $15 \mathrm{~m}$ de profundidad). A partir de la correlación de propiedades de la secuencia se demostró que la historia paleoambiental de los sedimentos lacustres determina el grado y tipo de cristalización de las arcillas, su evolución bajo diferentes regímenes hídricos, las heterogeneidades granulométricas de la secuencia y su historia de cargas en condiciones naturales. Se discute también la representatividad de los parámetros determinados con técnicas estandarizadas, límites de consistencia, cuando no se considera el tipo de agua retenida en la estructura de los materiales arcillosos. La relación entre las propiedades físicas y mecánicas de estos materiales es objeto de investigación de diferentes grupos de trabajo en México y países como Japón, Canadá e Inglaterra. Sin embargo, pocos estudios han integrado de manera sistemática los diversos factores geológicos que condicionan las propiedades y el comportamiento de los materiales sedimentarios.

Los estudios del fracturamiento en sedimentos fluviolacustres revelan la coexistencia de varios factores que determinan las características de diversos tipos de fracturas a diferentes escalas.

Frecuentemente el fracturamiento es estudiado en una sola escala y al intentar simplificar el fenómeno no es posible identificar los mecanismos que lo determinan ni sus condiciones de propagación. Los resultados presentados muestran que la adecuada utilización de la información geo-

Tabla 5: Comparación de índices de plasticidad (IP) para condiciones de humedad natural y de secado al aire (valores extremos resaltados). Variación en profundidad.

\begin{tabular}{cccc}
\hline Profundidad m & IP Húmedo & IP Seco & Disminución \% \\
\hline 1.50 & 147 & 20 & 86.4 \\
1.80 & 153 & 14 & $\mathbf{9 0 . 8}$ \\
2.50 & 49 & 20 & 59.2 \\
3.60 & 62 & 17 & 72.6 \\
7.25 & 98 & 49 & $\mathbf{5 0 . 0}$ \\
9.25 & 92 & 30 & 67.4 \\
11.8 & 124 & 33 & 73.4 \\
12.75 & 100 & 34 & 66.0 \\
\hline
\end{tabular}


lógica permite una mejor comprensión de los mecanismos de fracturamiento para cado caso de estudio y debe llevar a una evaluación más precisa del peligro que implican para la infraestructura urbana.

\section{Agradecimientos}

Los autores agradecen el apoyo recibido para esta investigación por el Centro de Geociencias de la UNAM para

Tabla 6: Propiedades mecánicas y clasificación SUCS de la secuencia estudiada, Chalco. Variaciones de compresibilidad en profundidad. $\sigma$, esfuerzo efectivo vertical; Pc, carga de pre-consolidación; Cc; índice de compresibilidad; ei-ef, diferencia de relación de vacíos inicial y final.

\begin{tabular}{llllll}
\hline $\begin{array}{c}\text { Prof. } \\
(\mathbf{m})\end{array}$ & Color SUCS & $\begin{array}{c}\boldsymbol{\sigma}_{\mathbf{v}}^{\prime} \\
\mathbf{k g} / \mathbf{c m}^{2}\end{array}$ & $\begin{array}{c}\text { Pc } \\
\mathbf{k g} / \mathbf{c m}^{2}\end{array}$ & Cc & ei-ef $\left(\mathbf{P}_{\text {ap }}\right)$ \\
\hline 1.84 & $\begin{array}{l}\text { Rojo oscuro grietas } \\
\text { OH-MH }\end{array}$ & 0.35 & 0.784 & $5.90-5.70$ \\
& & & & \\
2.44 & $\begin{array}{l}\text { Rojo oscuro } \\
\text { OH-MH }\end{array}$ & 0.09 & 0.54 & 2.139 & $4.20-3.70$ \\
3.75 & Negro OH-MH & 0.11 & 0.54 & 0.393 & $1.58-1.45$ \\
7.39 & $\begin{array}{l}\text { Pardo oscuro } \\
\text { OH-MH }\end{array}$ & $\mathbf{0 . 5 7}$ & 1.46 & $\mathbf{0 . 4 0 7}$ & $2.15-2.05$ \\
9.2 & & & & \\
11.9 & $\begin{array}{l}\text { Gris oliva CH } \\
\text { Gris oliva OH-MH }\end{array}$ & 0.17 & 2.08 & 1.299 & $2.35-2.05$ \\
12.7 & $\begin{array}{l}\text { Gris oliva } \\
\text { grietas }\end{array}$ & 0.28 & 1.991 & $3.70-3.30$ \\
& OH-MH & 0.66 & $\mathbf{2 . 4 2 2}$ & $4.50-4.10$ \\
14.8 & Gris oliva OH-MH 2.74 & 0.34 & 1.643 & $4.40-4.00$ \\
\hline
\end{tabular}

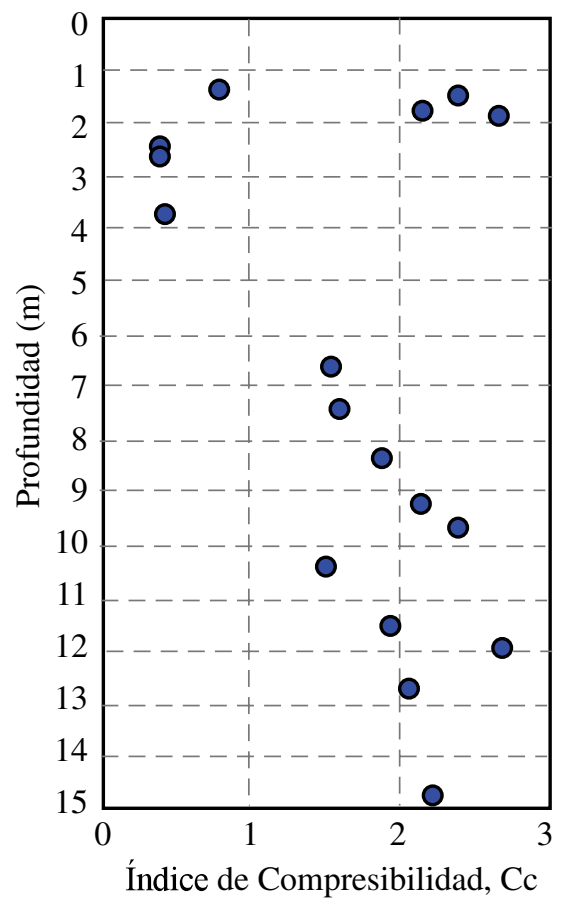

Figura 6. Variación con profundidad del índice de compresibilidad para la secuencia estudiada, Chalco. el desarrollo del proyecto interno investigación de Dora C. Carreón Freyre: "Análisis de subsidencia y fracturamiento en cuencas fluvio-lacustres. Estudios multidisciplinarios en valles del centro de México". También se agradece el apoyo del Sr. Ricardo Carrizosa para la caracterización de materiales en el laboratorio de Geomecánica del Centro de Geociencias. Asimismo, se agradece a la Química Patricia Girón, por las determinaciones de mineralogía de arcillas en el Laboratorio de Fluorescencia de Rayos X del Instituto de Geología de la UNAM.

\section{Referencias bibliográficas}

ASTM D4318-95a, 98. Standard test method for Liquid Limit, Plastic Limit, and Plasticity Index of soils. merican Society for Testing and Materials.

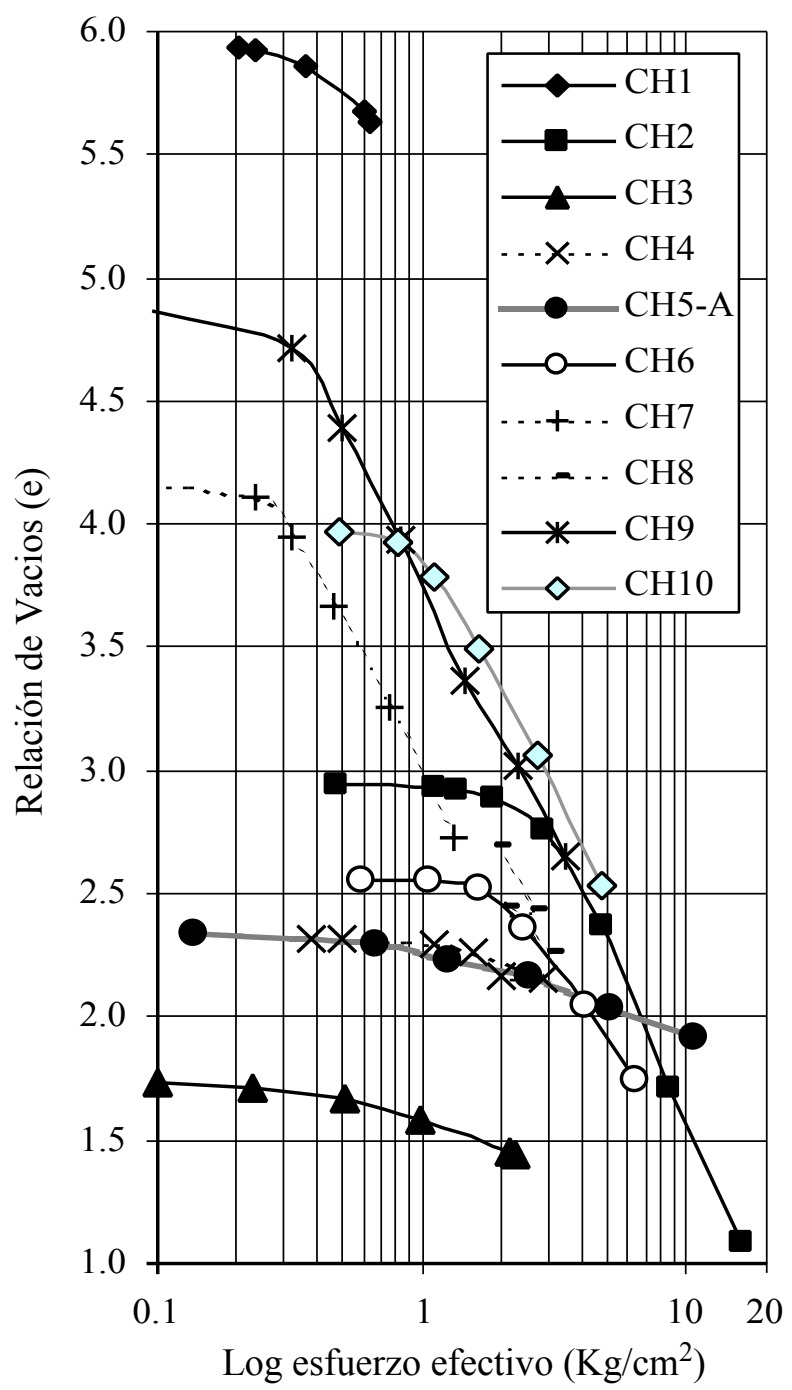

Figura 7. Curvas de compresibilidad e-log $\sigma$ para 10 muestras de diferentes profundidades de la secuencia estudiada, Chalco: $\mathrm{CH} 1-1.4 \mathrm{~m}, \mathrm{CH} 2-1.8$ m, CH3-2.44 m, CH4-2.64 m, CH5-3.75 m, CH6-7.24 m, CH7-9.2 m, CH8-9.71 m, CH9-12.7m, CH10-14.75 m. 
ASTM D2435-96, 1998. Standard test method for one-dimensional consolidation properties of soils. American Society for Testing and Materials.

Álvarez-Manilla Aceves., A., 2000. Modelo del mecanismo de agrietamiento en el Valle y zona metropolitana de Querétaro. Tesis de maestría. Universidad Autónoma de Querétaro.

Auvinet, G. y Arias, A., 1991. Propagación de grietas. En "Agrietamiento de Suelos". Ed. Sociedad Mexicana de Mecánica de Suelos, 2131.

Alberro, J. y Hernández. R., 1990. Génesis de las grietas de tensión en el Valle de México. En "El subsuelo de la cuenca del Valle de México y su relación con la ingeniería de cimentaciones a cinco años del sismo". Sociedad Mexicana de Mecánica de Suelos, 95-106.

Caballero, M. y Ortega-Guerrero, B., 1998. Lake levels about 40,000 years ago at Lake Chalco, near Mexico City. Quaternary Research 50, 69-79.

Carreón-Freyre, D., Cerca, M. 2006. Delineating the near-surface geometry of the fracture system affecting the valley of Queretaro, Mexico: Correlation of GPR signatures and physical properties of sediments. Near Surface Geophysics, EAGE (European Assoc. of Geoscientists and Engineers). 4(1): 49-55.

Carreón-Freyre, D. C., 2005. Importancia de la caracterización de arcillas en laboratorio para la adecuada evaluación de sus propiedades mecánicas, pp. 273-282. En Bucio, L., Cristalografia: Fundamentos Técnicas y Aplicaciones, Sociedad Mexicana de Cristalografia. A. C., ISBN 970-9888-07-2, $528 \mathrm{p}$.

Carreón-Freyre., D., Cerca Martínez, M. y Hernández Marín, M., 2005a. Propagation of fracturing related to land subsidence in the Valley of Queretaro, Mexico. Proceedings of the $7^{\text {th }}$ International Symposium on Land Subsidence SISOLS 2005, Shanghai, P.R. China.. ISBN 7-5323-8209-5. Vol. I: 155-164.

Carreón-Freyre., D., Cerca M., Luna-González, L., Gámez-González, F. J., 2005b. Influencia de la estratigrafía y estructura geológica en el flujo de agua subterránea del Valle de Querétaro. Revista Mexicana de Ciencias Geológicas 22(1): 1-18.

Carreón-Freyre., D.C., Cerca M., Hernández-Marín, M., 2003. Correlation of near-surface stratigraphy and physical properties of clayey sediments from Chalco Basin, Mexico, using Ground Penetrating Radar. Journal of Applied Geophysics 53: 121-136.

Carreón-Freyre, D.C., Gama-Castro, J., Palacios-Mayorga, S., GarnicaAnguas, P., 1998. Propiedades y clasificación de los suelos residuales de México. Memorias de la XIX Reunión Nacional de Mecánica de Suelos, 75-80.

Carrillo, N., 1947. Influence of artesial wells in the sinking of Mexico City, en Volumen Nabor Carrillo "El hundimiento de la Ciudad de México y el Proyecto Texcoco". Comisión Impulsora y Coordinadora de la Investigación Científica Anuario 47: 7-14.

De Pablo Galán, L., De Pablo, J. J., Chávez Garcia, L., 2001. Diagenesis and shear rheology of a Recent-Pleistocene volcanogenic sequence, Mexican Basin. Implication ti swelling and stability. Revista Mexicana de Ciencias Geológicas 18(2): 175-185.

Díaz-Rodríguez, A., Lozano-Santacruz, R., Dávila-Alcocer, V.M., Vallejo, E and Girón, P., 1998. Physical, chemical, and mineralogical properties of México City sediments: a geotechnical perspectiva. Can. Geotech.J. 35, 600-610.

Díaz-Rodríguez, A., and Santamarina, J.C., 2001. Mexico City soil behavior at different strains: Observations and physical interpretation. J. of Geotechnical and Geoenvironmental Engineering 127 (9), 783789.

Ellstein, A. 1978. Teoría sobre el mecanismo de falla. El subsuelo y la ingeniería de cimentaciones en el área urbana del valle de México, SMMS, 177-181.

Figueroa Vega, G. E., 1989. Mecanismos de producción de grietas inducidos por la explotación del agua subterránea. Academia Mexicana de Ingeniería. Alternativas Tecnológicas 29, 371-378.

Gama Castro .J. E., Carreón Freyre .D.C., Palacios M. S., Solleiro Rebolledo .E., 1998, Guía para la Identificación de Suelos Residuales. Aplicación a la Construcción de Vías Terrestres. Ed. Instituto Mexicano del Transporte, $156 \mathrm{p}$.

Gutiérrez Castorena, M. del C., Stoops, G., Ortiz Solorio, C. A., López
Avila, G. L., 2005. Amorphous silica materials in soils and sediments of the Ex-Lago de Texcoco, México: An explanation for its subsidence. Catena 60: 205-226.

Hernández Marín, M. y Carreón-Freyre., D., 2002. Análisis de variación de la presión de poro durante pruebas de consolidación incrementales en suelos vulcano lacustres. Memorias de la XXI Reunión Nacional de Mecánica de Suelos. 1: 3-12. ISBN 968-5350-09-4.

Hiriart, F., Marsal, R. J., Cruickshank, G. y Key, F., 1952. Contribución de la comisión Federal de Electricidad a la solución del problema de abastecimiento de agua a la Ciudad de México. Comisión Federal de Electricidad.

Holzer, T. L. and Davis, S. N., 1976. Earth fissures associated with watertable declines (abs.). Geological Society of America: 8(6): 923-924.

Holzer, T. L., 1984. Ground failure induced by ground-water withdrawal from unconsolidated sediment. Geological Society of America. Reviews in Engineering Geology VI: 67-105.

Juárez Badillo, E., 1962. Mecanismos de grietas de tensión en el Valle de México. Tesis doctoral, UNAM. México.

Juárez Badillo., E. y Figueroa Vega, G. E., 1984. Stresses and displacements in an aquifer due to seepage forces (one dimensional case). Journal of Hydrology 73: 259-288.

Juárez Badillo., E., 1991. Grietas por fuerzas de filtración. "Agrietamiento de Suelos". Sociedad Mexicana de Mecánica de Suelos, 39-42.

Juárez Luna, G., Rangel Nuñez, J. L., Ayala Milán A. G., Romo Organista, M. P., 2002. Modelado del fracturamiento en las arcillas del Valle de México. Memorias de la XXI Reunión Nacional de Mecánica de Suelos 1: 183-192. ISBN 968-5350-09-04.

Kreitler, Ch. W., 1976. Faulting and land subsidence from groundwater and hydrocarbon production, Houston-Galveston, Texas, U.S.A. Second IASH, California. No. 121, 435.

Kreitler, C. W.,1977. Fault control of subsidence, Houston, Texas. Ground Water 15 (3): 203-214.

Larson, M. K., 1984. Potential for subsidence fissuring in the Phoenix Arizona USA area. Third IAHS. Venice, Italy. No. 151: 291-299.

Lozano-García, M.S., Ortega-Guerrero, B., Caballero-Miranda, M., and Urrutia-Fucugauchi, J., 1993. Late Pleistocene and Holocene paleoenvironments of Chalco Lake, Central Mexico. Quaternary Research 40, 332-342.

Lugo Hubp J., Pérez Vega A., Rojas Salas M., 1991. Formación de grietas en la margen del antiguo lago al oriente de la cuenca de México. Geofísica Internacional, 30 (2): 87-95.

Marsal, R. J. y Mazari, M. 1959. El subsuelo de la Ciudad de México. Instituto de Ingeniería. U.N.A.M.

Mazari, M. y Alberro, J. 1990. Hundimiento de la Ciudad de México. En "Problemas de la Cuenca de México", coordinado por J. Kumate y M. Mazari. Ed. El Colegio Nacional. pp 83-114.

Mazari-Hiriart, M, Hernández-Eugenio, C., Rojo-Callejas, F., LozanoSantacruz, R., 2000. Vertical variability of PCE sorption in the lacustrine clays of Mexico City. Environmental Geology 39(6), 595-602.

Mesri G., Rokhse A. y Bonor B. F., 1976. Compositive and compressibility of typical samples of Mexico City, Geotechnique 25(3), 527-554.

Mooser, F. 1975. Historia geológica de la Cuenca de México. En "Memorias de las Obras del Sistema de Drenaje Profundo del Distrito Federal". DDF. Tomo I: 7-38.

Ohtsubo, M., Takayama, M. y Egashira, K., 1983. Relationships of consistency limits and activity to some physical and chemical properties of Ariake marine Clays. Soils and foundations. 23 (1): $38-46$.

Orozco, J. M. y Figueroa V., G., 1991. Descripción cronológica del desarrollo de los conocimientos sobre el agrietamiento de terrenos. Agrietamiento de suelos, Soc. Mex. Mec. de Suelos, 1-12.

Peralta y Fabi, R., 1989, Sobre el origen de algunas propiedades mecánicas de la formación arcillosa superior del Valle de México. Simposio sobre Tópicos Geológicos de la Cuenca del Valle de México.

Poland, J. F., 1984. Guidebook to studies of land subsidence due to groundwater withdrawal. UNESCO. $304 \mathrm{p}$.

Rojas González, E., Arzate Flores, J., Arroyo, M., 2002. A method to predict the group fissuring and faulting caused by regional 
groundwater decline. Engineering Geology, 65, 245-260.

Sandoval, J. P., and Bartlett, S.R., 1991. Land subsidence and earth fissuring on the central Arizona Project. IAHS. Houston, Texas. No. 200: 249-260.

Saarenketo, T., 1998. Electrical properties of water in clay and silty soils. J. of Applied Geophysics 40: 73-88.

Schumann, H.H., and Poland, J. F., 1969. Land Subsidence, Earth Fissures and Groundwater withdrawal in South-Central Arizona, U.S.A. First Symposium IASH-UNESCO, Tokio, 295-302.

Trejo M., A. y Baini M., A., 1991. Agrietamiento de suelos en la zona de Querétaro. En "Agrietamiento de Suelos". SMMS, 67-74.

Trujillo Candelaria, J. A., 1989. Fallamiento de terrenos en Celaya, Gto. Academia Mexicana de Ingenieria. Alternativas Tecnológicas 29, 367-369.

Tuckwell, G. W., Lonergan, L, and Jolly, R. J., 2003. The comtrol of stress history and flaw distribution on the evolution of polygonal fracture networks. J. of Structural geology 25: 1241-1250.

Velde, B., 1995. Origin and Mineralogy of Clays. Clays and the environment. Springer-Verlag.

Velde, B., Composition and mineralogy of Clay Minerals, 8-41.

Righi, D. and Meunier, A., Origin of Clays by rock weathering and soil formation, 43-157.
Hillier, S., Erosion, sedimentation and sedimentary origin of clays, $162-214$.

Wada, K., 1987. Minerals formed and mineral formation from volcanic ash by weathering. Chemical Geology 60, 17.28.

Warren, C.J., and Rudolph, D.L., 1997. Clay minerals in basin of Mexico lacustrine sediments and their influence on ion mobility in groundwater. Journal of Contaminant Hydrology 27, 177-198.

Wesley, L.D., 2001. Consolidation behaviour of allophane clays. Géotechnique 51(10), 901.904.

Zeevaert, L., 1953. Estratigrafía y problemas de ingeniería en los depósitos de arcilla lacustre de la Ciudad de México. Memoria del Congreso Científico Mexicano Vol. 5: 58-70.

Manuscrito recibido: Noviembre 15, 2006

Manuscrito corregido recibido: Enero 8, 2007

Manuscrito aceptado: Febrero 1, 2007 